\title{
Solution of the boundary value problem for optimal escape in continuous stochastic systems and maps
}

\author{
S. Beri, ${ }^{1}$ R. Mannella,${ }^{2,1}$ D. G. Luchinsky, ${ }^{1}$ A. N. Silchenko, ${ }^{1}$ and P. V. E. McClintock ${ }^{1}$ \\ ${ }^{1}$ Department of Physics, Lancaster University, Lancaster LA1 4YB, United Kingdom \\ ${ }^{2}$ Dipartimento di Fisica, Università di Pisa and INFM UdR Pisa, Largo Pontecorvo 3, 56127 Pisa, Italy
}

(Received 15 March 2005; published 29 September 2005)

\begin{abstract}
Topologies of invariant manifolds and optimal trajectories are investigated in stochastic continuous systems and maps. A topological method is introduced that simplifies the solution of boundary value problems: The activation energy is calculated as a function of a set of parameters characterizing the initial conditions of the escape path. The method is applied explicitly to compute the optimal escape path and the activation energy for a variety of dynamical systems and maps.
\end{abstract}

DOI: 10.1103/PhysRevE.72.036131

PACS number(s): 02.50.Ey, 05.40.Jc

\section{INTRODUCTION}

The understanding of activation processes in nonlinear stochastic maps and flows is one of the long-standing problems of statistical physics. It attracts much attention in diverse scientific contexts, e.g., in relation to stochastic resonance [1], directed diffusion in stochastic ratchets [2-4], nucleation in electrochemical systems [5], the dynamics of VCSELs [6-9] and gas lasers [10,11], and the passage of ions through open ionic channels [12] in biological membranes. From a mathematical point of view, the interest in activation problems derives in large part from the many stimulating topological discoveries that have been made in such systems [13-19].

The most promising steps toward a solution have been achieved in the regime of small noise intensities. In this asymptotic condition, the activation process can be described using a nonequilibrium potential (or equivalently an activation energy). The calculation of the activation energy requires the minimization of a "cost functional" $S(x, t)$ along a family of trajectories and the calculation of an optimal escape path that the system follows during the activation process with overwhelming probablity in the limit of zero noise intensity $[5,20-24]$.

For a general system, the "potential" $S(x, t)$ is a multivalued function of position $x$ in the system state space $[4,5,15,20,25-34]$. Its calculation requires a topological analysis of the action surface $[19,27,31,35]$ and minimization over the set of all trajectories emanating from the initial steady state and terminating at $x$. The trajectory providing the escape from a basin of attraction with least cost is known as the most probable escape path (MPEP) $[15,22,32]$. It is a heteroclinic trajectory lying on the intersection of the unstable manifold of the initial state and the stable manifold of the boundary.

In general, the structure of the manifolds can be wildly singular and many heteroclinic trajectories may exist. The location of the MPEP is consequently a very difficult task: Many almost degenerate local minima appear in the action, and under these conditions the standard methods of minimization do not provide a reliable means of extracting the global minimum. The multivaluedness of the potential at the boundary makes minimization almost impossible to achieve in practice for escape from chaotic attractors [36], or in activation problems for systems where the basin boundaries are fractal $[37,38]$, or when the escape rate is enhanced by the presence of a chaotic saddle embedded in the basin [39].

In this paper we present a method of solving boundary value problems which significantly simplifies the investigation in all of the difficult scenarios mentioned above. The same formalism applies both to maps and to continuous systems. It is shown that all the results obtained for a map can be extended to continuous systems by taking the appropriate limit; quite counter-intuitively, however, not all of the results available for continuous systems can be exported to maps. We treat noise-activated escape in two-dimensional systems exhibiting unstable cycles, and in two-dimensional metastable and multistable maps.

In Sec. II we outline the Hamiltonian theory of large fluctuations in maps and introduce the MPEP, cost function, and the nonequilibrium potential or quasipotential. Section III iscusses singularities in the quasipotential including caustics, cusp points, and switching lines, taking as an example the inverted van der Pol oscillator (IVDP). A derivation of the boundary conditions is presented in Sec. IV. Section V provides a linear analysis of the action surface and applies it to a simple 1D system, taken as an example. The parametrization of the family of trajectories is discussed in Sec. VI. What we will refer to as the action plot is introduced in Sec. VII. It represents an easy way to relate the topological properties in the phase space to the features of the nonequilibrium potential. Its self-similar properties are discussed, and it is then applied in turn to a series of examples: The IVDP, the harmonically driven overdamped Duffing oscillator, and the Henon and Julia maps. In all cases the theory is tested by numerical simulations. Finally, Sec. VIII summarizes the results and draws conclusions.

\section{THEORY}

A stochastic map is a discrete dynamical system with a stochastic term. In this paper we consider the very general case of a Markovian system described by a map of the form 


$$
x_{n+1}=K\left(x_{n}\right)+\sigma \xi_{n} .
$$

Here $\left\{x_{n}\right\}$ a set of coordinates describing the system, $K(x)$ is a nonlinear function, and $\left\{\xi_{n}\right\}$ is a vector of stochastic variables mixed by the noise matrix $\sigma$. The stochastic variables $\xi_{n}$ are assumed to be Gaussianly distributed with

$$
\left\langle\xi_{n}\right\rangle=0 \forall n \quad\left\langle\xi_{n} \xi_{m}\right\rangle=\epsilon \delta_{n m},
$$

where $\epsilon$ is the noise intensity. In the rest of the paper, without loss of generality in the results the noise matrix $\sigma$ is assumed to be the identity matrix.

A continuous system of the form $\dot{x}=K(x)+\xi(t)$ can be written as the limit for $h \rightarrow 0$ of the map $x_{n+1}=x_{n}+h K\left(x_{n}\right)$ $+z_{n}$, where the new Gaussian variable $z_{n}=\int_{0}^{h} \xi(t) d t$ has the momenta $\langle z\rangle=0$ and $\langle z(t) z(s)\rangle=\epsilon h \delta(t-s)$. For this reason, in what follows, we will focus on the more general case of stochastic maps of the form (1) and we will recover the results for continuous systems using a limit procedure. The function $K(x)$ in Eq. (1) is chosen such that the system displays coexisting stable orbits.

When the noise intensity $\epsilon$ is small, the system is expected to spend most of its time in the vicinity of one of the stable states: Only occasionally will it move away from the stable state by a distance larger that $\sqrt{\epsilon}$.

The probability of a transition from the initial point $x_{i}$ to a final point $x_{f}$ with $\left|x_{f}-x_{i}\right|>\sqrt{\epsilon}$ can be expressed as

$$
P\left(x_{f}, x_{i}\right)=\int_{\gamma} P[\gamma] d\{\gamma\} .
$$

Here the integration is taken along all possible paths $\gamma$ joining the initial and final states and the functional $P[\gamma]$ expresses the probability of the system fluctuating along the path $\gamma$. For a stochastic map of the form (1), and a noise described by Eq. (2), the probability $P[\gamma]$ for a path $\gamma$ $=\left\{x_{1} \cdots x_{N}\right\}$ can be written as

$$
P[\gamma] \propto \exp \left[-\frac{S\left[x_{1} \cdots x_{N}\right]}{\epsilon}\right] .
$$

The function $S\left[x_{1} \cdots x_{N}\right]$ is a cost function defined as

$$
S\left[x_{1} \cdots x_{N-1}\right]=\frac{1}{2} \sum_{n=0}^{N-1} \xi_{n}^{2}
$$

with the constraint between $x_{n}$ and $\xi_{n}$ expressed by Eq. (1).

In the asymptotic limit $\epsilon \rightarrow 0$, the transition probability (3) is dominated by the contribution from the path that minimizes the cost $S[\gamma]$. The contributions of other paths to the probability are exponentially suppressed. In other words, when $\epsilon \rightarrow 0$, it is less and less likely that a transition will occur, but, on the other hand, when such an event manifests, it is exponentially likely to be according to the path that minimizes the cost.

The probability of the transition takes the asymptotic form

$$
P\left(x_{N} \mid x_{0}\right)=z \exp \left[-\frac{S_{\min }}{\epsilon}\right],
$$

where $S_{\min }$ is the least cost for the transition. The preexponential factor (or prefactor) gives a correction to the escape probability related to the number of paths close to the path with least cost [40].

When the activation problem is considered explicitly, the initial state $x_{i}$ must be taken in the vicinity of one of the stationary states, and the final state $x_{f}$ has to be chosen on the boundary of the basin of attraction of the stable structure. The path which realizes the escape with the least cost is known as the most probable escape path (MPEP). In order to solve the escape problem is necessary to work out the MPEP and calculate the cost along it. In this way, the probability of escape can be calculated using Eq. (6). The solution will be asymptotically correct in the limit $\epsilon \rightarrow 0$.

The cost function $S$ introduced in Eq. (5) has to be minimized with respect to the set of all possible noise bursts $\left\{\xi_{n}\right\}$ and to the set of all possible intermediate coordinates $\left\{x_{n}\right\}$. It is clear that Eq. (1) gives a constraint between the values of $\left\{x_{n}\right\}$ and $\left\{\xi_{n}\right\}$. The constraint is taken into account by performing the minimization using the method of Lagrange undetermined multipliers. The auxiliary cost function

$$
\tilde{S}=\frac{1}{2} \sum_{n}^{N} \xi_{n}^{2}+\lambda_{n}\left[x_{n+1}-K\left(x_{n}\right)-\xi_{n}\right]
$$

is introduced, where $\left\{\lambda_{i}\right\}$ is a set of Lagrangian multipliers. The auxiliary function $\tilde{S}$ must be minimized with respect to $\xi_{i}, x_{i}, \lambda_{i}$ as independent variables. The following relationships are obtained from the minimization:

$$
\begin{gathered}
x_{n+1}=K\left(x_{n}\right)+\lambda_{n}, \\
\lambda_{n+1}=\left[\left.\frac{\partial K}{\partial x_{i}}\right|_{x_{n+1}}\right]^{-1} \lambda_{n} .
\end{gathered}
$$

Equations (8) define an extended area-preserving map. The cost function can be considered as evolving along the trajectories according to

$$
S_{n+1}=S_{n}+\frac{1}{2} \lambda_{n}^{2}
$$

The calculation of the transition probability can be now performed using Eq. (8) to calculate the evolution of the system, combined with Eq. (9).

For a continuous system, the limit $h \rightarrow 0$ gives

$$
\begin{aligned}
& \dot{x}=K(x)+\lambda, \\
& \dot{\lambda}=-\left[\frac{\partial K}{\partial x}\right] \lambda .
\end{aligned}
$$

Equations (10) and (11) can be seen as a set of equations describing an auxiliary Hamiltonian system. The Lagrange multiplier $\lambda$ plays the role of a momentum $p$ conjugate to the coordinate $x$. The Hamiltonian for this system is known as the Wentzell-Freidlin Hamiltonian

$$
H(x, \lambda)=\frac{1}{2} \lambda^{2}+\lambda K .
$$

Considering the formal Hamiltonian structure of Eqs. (10) and (11), when discussing continuous systems, we will refer 
to the Lagrangian multipliers as momenta and we will use the notation $\lambda$ or $p$ independently.

The equation for the evolution of the cost function along Eqs. (10) and (11) is $S_{i+1}=S_{i}+\frac{1}{2} h \lambda_{i}^{2}$; in the limit $h \rightarrow 0$ it reads as

$$
\dot{S}=\frac{1}{2} \lambda^{2}=\frac{\partial H}{\partial \lambda} \lambda-H .
$$

$S$ evolves according to the equation for a classical action in the system described by the Hamiltonian (12). Hamiltonian systems (10) and (11), Hamiltonian (12), and Eq. (13) for the cost function, can all be obtained in an alternative way through asymptotic analysis of the Fokker-Planck equation $[19,41]$.

Generally, there are infinitely many solutions of Eqs. (8) which emanates from the initial state $x_{0}$ and reach the final state $x_{N}$; each of them having a corresponding cost. They correspond to local minima of the cost function; however, in the limit of small noise intensity, the probability density for the system is dominated by the global minimum of the action, because the contributions of the other local minima become exponentially small. This property has been confirmed by analog experiments $[42,43]$ : The ensemble average of a large number of trajectories from an initial stationary point to a final one is found to be consistent with the least cost trajectory, and not with other local minima. In other words, only those trajectories corresponding to a global minimum can be observed in a physical experiment in the zero noiseintensity limit. For this reason, these trajectories are called physical and the ones corresponding to local minima unphysical [18].

The probability distribution is then defined by the minimum value of the cost $S_{\min }(x)=\min \left\{S_{m}(x)\right\}$, where $S_{m}$ are the costs calculated along different trajectories terminating in $x$. All the functions $S_{m}$ are smooth, but the procedure of minimization might produce nondifferentiability in $S_{\text {min }}$ $[25,26,28]$. For nonequilibrium systems $S_{\min }$ is continuous, but it is not everywhere differentiable. The nondifferentiability of the cost function is a generic feature of nonequilibrium systems and has been intensively investigated $[1,18,25,26,28]$. Although not differentiable everywhere, nevertheless the function $S_{\min }$ cannot be arbitrarily irregular. First, $S$ is nondecreasing along the trajectories of Eq. (8). Second [44], $S_{\min }$ is locally a Lipschitz function; i.e., for every point $x_{0}$ in the coordinate space, a neighborhood of $x_{0}$ exists such that for any pair of points $x_{1}$ and $x_{2}$

$$
\left|S_{\min }\left(x_{1}\right)-S_{\min }\left(x_{2}\right)\right|<L\left|x_{1}-x_{2}\right|,
$$

where $L$ is a finite constant. The local Lipschitz condition is enough to imply differentiability almost everywhere [28].

It is known from the theory of nondifferentiable Lyapunov functions that, if $S_{\min }$ is nondecreasing along the trajectories of Eq. (8) and it is locally a Lipschitz function, then all theorems valid in the differentiable case can be generalized [28]. This allows the use of $S_{\min }$ as a nonequilibrium potential (or quasipotential) for the system.

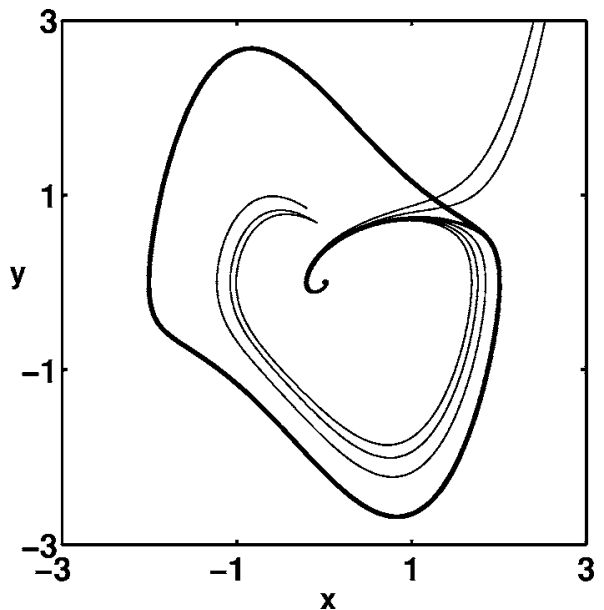

FIG. 1. Structure of the escape trajectories for the IVDP system. Here the origin is a stable point for the noise-free dynamics and the cycle (bold line) is an unstable limit cycle. The MPEP is shown as a thick line. It reaches the limit cycle asymptotically with no momentum. Some trajectories that are small perturbations of the MPEP are also shown (thin lines). They may either cross the cycle or be reflected, moving back to the initial state. The MPEP separates these two families of trajectories.

\section{TOPOLOGY}

The presence of singularities in the quasipotential $S_{\min }$ is related to the presence of singular features in the pattern of solutions of Eq. (8) or Eqs. (10) and (11). They provide one of the main difficulties in the solution of the boundary values problem.

We consider the case of a continuous planar system presenting a saddle cycle $S_{c}$ coexisting with a stable equilibrium point $x_{0}$. The escape problem is formulated by setting the following boundary value conditions:

$$
\lim _{t \rightarrow-\infty} x=x_{0} \quad \lim _{t \rightarrow+\infty} x=S_{c}
$$

The picture for a two-dimensional map is similar.

It is known from the theory of dynamical systems [45] that the trajectories providing solutions of Eq. (11) emanating from $x_{0}$ for $t \rightarrow-\infty$ span a two-dimensional Lagrangian unstable manifold (UM) in the four-dimensional phase space. The projections of these trajectories onto the coordinate space determine the pattern of escape trajectories. If we follow the motion of these trajectories, we expect three different behaviors, as summarized in Fig. 1, where the behavior of escape trajectories is shown for an inverted van der Pol (IVDP) system. It is an example of an autonomous system with a stable point coexisting with a saddle cycle described by the following equation:

$$
\ddot{x}+2 \eta\left(1-x^{2}\right) \dot{x}+x=0,
$$

where $x$ is a dynamical variable and $\eta>0$ is real coefficient. In the coordinate space $x, y=\dot{x}$, the noise drives the system from the stable point $(0,0)$ toward the saddle cycle according to the solutions of Eqs. (10) and (11). Some of the trajectories cross the cycle and continue their fluctuational motion indefinitely. Some other trajectories have insufficient mo- 
mentum $\vec{p}$ to overcome the repelling force of the cycle and they are reflected back toward the initial stable state. A particular trajectory separates the two families: It reaches the saddle cycle asymptotically (for $t \rightarrow \infty$ ) and tangentially ( $p$ $\rightarrow 0$ for $t \rightarrow 0$ ). In the extended phase space, such a trajectory lies on the intersection of the unstable manifold on the initial state and the stable manifold of the final cycle. The correct mathematical definition for a path having such a property is a heteroclinic trajectory.

It is classical result of the asymptotic theory of activation $[15-17,19,26,31]$ that the most probable escape path must be a heteroclinic trajectory.

In a nonequilibrium system with saddles, the structure of both manifolds and patterns of trajectories can be singular $[13,15,17,19]$. The trajectories solution of Eq. (11) may present multiple intersections in the coordinate space, and the pattern presents singular features such as caustics and cusp points. Caustics appear in the pattern as envelopes of trajectories; the cusps correspond to the merging of two separate branches of a caustic. A caustic can be generated by a family of trajectories during their motion toward the saddle (where $\lambda$ is substantially different from zero) or by trajectories which are relaxing back toward the stable state (where $\lambda$ is near zero). Since the momentum of a trajectory becomes exponentially small as it approaches the saddle, the closer a trajectory gets to the limit cycle before being reflected, the more it resembles a purely relaxational trajectory during its motion toward the initial state and the contact with the caustics takes place far inside the cycle. For this reason, the internal branch of the caustic always converges to the initial state.

In the region included by caustics, the escaping trajectories intersect those trajectories that are reflected back. Such apparent intersection results from projection onto the coordinate space, as the Cauchy theorem does not allow the trajectories to cross in the extended phase space and the "crossing" paths must have different values of momenta. In these regions, the Lagrangian manifold is threefold. What appears to be an intersection occurs between trajectories on different sheets of the manifold. A trajectory starts on one of the two external leaves of the fold. Then it eventually moves to the central leaf. In the projection, the leaf swap appears as a reflection by a caustic $[17,19]$. Once a trajectory is on the central leaf of the manifold, it stays there forever, because any further contact with the caustics is forbidden by the Cauchy theorem. Following contact with a caustic, the dynamics of a trajectory continue on the central leaf of the manifold, and each of the trajectories on the central leaf may present the same behavior as the trajectories on the external leaves. In particular, heteroclinic trajectories other than the MPEP are present on the central leaf. Considering the pattern of trajectories on the central leaf of the manifold, it may present intersections similar to those observed for the full pattern. This means that the central sheet is folding again generating new caustics and cusps. This pictures repeats again and again in a self similar way.

In the region where different trajectories intersect, the action is multivalued. In the limit $\epsilon \rightarrow 0$, only the trajectories reaching a point $x$ with least action can be observed experimentally. A continuous line separates the regions where the physical trajectories are qualitatively different. Such a line is called the switching line (SL) $[17,19]$. Each point on the SL is reached by two different trajectories having exactly the same action. In the example of the IVDP, the SL separates the region where the physical trajectories have a momentum $\lambda$ significantly different from zero from the regions where the physical trajectories are relaxing back toward the internal part of the cycle (having $\lambda \sim 0$ ). On the action surface, the switching line corresponds to the intersection (at a nonzero angle) of two sheets of the action surface. Cutting off the nonphysical parts of the action surface (i.e., those with nonminimum action), corresponding to the switching lines, we observe a nondifferentiability of the surface: The action is continuous on the SL, but the first derivative in the orthogonal direction switches abruptly between two different finite values.

\section{BOUNDARY CONDITIONS}

In the previous sections, the complex, self-repeating, singular structure of the manifold has been illustrated. All these are global properties, related to the nonlinear form of $K(x)$ and to the lack of detailed balance in the system. In order to set the boundary conditions properly, however, the shape of the manifold in the vicinity of the stationary states must be known.

In this section, we investigate the topology of the Lagrangian manifolds in the vicinity of stationary points, for both maps and continuous systems, using linear analysis. We start from the simple case of a period-one stationary orbit $x_{s}$ for a two-dimensional map. In the close vicinity of $x_{s}$, the extended map (8) is rewritten as

$$
\begin{gathered}
\delta x_{n+1}=A \delta x_{n}+\lambda_{n}, \\
\lambda_{n+1}=A^{-1 T} \lambda_{n},
\end{gathered}
$$

where $\delta x$ is the displacement from $x_{s}$ and $A$ is the Jacobian matrix of the original map calculated in $x_{s}$. The point $\delta x=0$; $\lambda=0$ is a stationary point of map (17).

The unstable manifold of $x_{s}$ is two dimensional and it is spanned by the two unstable eigenvectors of Eq. (17). Assume first that the eigenvalues $\alpha_{1}$ and $\alpha_{2}$ of $A$ are real and satisfy $\left|\alpha_{1,2}\right|<1$. The point $x_{s}$ is a stable node for the original map and a saddle point for the extended map (17). The eigenvalues for the extended map are $\alpha_{1}, \alpha_{2}, \alpha_{1}^{-1}, \alpha_{2}^{-1}$. The stable eigenvectors associated with the contracting eigenvalues are called $e_{s 1}$ and $e_{s 2}$ where the index $s$ indicates stable. The unstable eigenvectors are denoted by $e_{u 1}$ and $e_{u 2}$. As the point is a stable focus for the original system, the two contracting eigenvectors have no $\lambda$ components and the stable manifold coincides with the coordinate plane. On the other hand, the expanding eigenvectors must have $\lambda$ components different than zero.

A generic point $x_{0}=(\delta x, \lambda)$ in the neighborhood of $\left(x_{s}, 0\right)$ can be written as a linear combination of the eigenvectors

$$
x_{0}=c_{s 1} e_{s 1}+c_{s 2} e_{s 2}+c_{u 1} e_{u 1}+c_{u 2} e_{u 2},
$$

where the coefficients $c_{s 1}, c_{s 2}, c_{u 1}$, and $c_{u 2}$ are real. The evolution of the point $x_{0}$ is 


$$
x_{0}=c_{s 1} \alpha_{1}^{n} e_{s 1}+c_{s 2} \alpha_{2}^{n} e_{s 2}+c_{u 1} \alpha_{1}^{-n} e_{u 1}+c_{u 2} \alpha_{2}^{-n} e_{u 2} .
$$

It is clear that the components of $x_{n}$ along $e_{s 1,2}$ shrink to zero and the components along $e_{u 1,2}$ expand. The eigenvectors $e_{u 1,2}$ span the unstable manifold. Once $e_{u 1,2}$ are known, an expression for the Lagrangian manifold can be obtained: The generic form for a point on the UM is obtained by setting $c_{s 1}=c_{s 2}=0$ in Eq. (18). Separating the space and the $\lambda$ components we obtain

$$
\left(\begin{array}{l}
\vec{x} \\
\vec{\lambda}
\end{array}\right)=c_{u 1}\left(\begin{array}{l}
\vec{e}_{u 1 x} \\
\vec{e}_{u 1 \lambda}
\end{array}\right)+c_{u 2}\left(\begin{array}{l}
\vec{e}_{u 2 x} \\
\vec{e}_{u 2 \lambda}
\end{array}\right) .
$$

Here $\vec{u}_{u 1 x}$ and $e_{u 1 \lambda}$ represent the $x$ and $\lambda$ components of the eigenvectors $\vec{e}_{u 1,2}$. Writing the vectors $x$ and $\lambda$ explicitly in terms of their components $x, y, \lambda_{x}, \lambda_{y}$, a set of four linear equations is obtained

$$
\begin{aligned}
\left(\begin{array}{c}
x \\
y
\end{array}\right) & =\left(\begin{array}{ll}
e_{u 1 x} & e_{u 2 x} \\
e_{u 1 y} & e_{u 2 y}
\end{array}\right)\left(\begin{array}{l}
c_{u 1} \\
c_{u 2}
\end{array}\right) \\
\left(\begin{array}{c}
\lambda_{x} \\
\lambda_{y}
\end{array}\right) & =\left(\begin{array}{ll}
e_{u 1 \lambda_{x}} & e_{u 2 \lambda_{x}} \\
e_{u 1 \lambda_{y}} & e_{u 2 \lambda_{y}}
\end{array}\right)\left(\begin{array}{c}
c_{u 1} \\
c_{u 2}
\end{array}\right) .
\end{aligned}
$$

Using standard linear algebra techniques, a linear relation among $\vec{x}$ and $\vec{\lambda}$ s may be obtained

$$
\left(\begin{array}{c}
\lambda_{x} \\
\lambda_{y}
\end{array}\right)=\left(\begin{array}{ll}
e_{u 1 \lambda_{x}} & e_{u 2 \lambda_{x}} \\
e_{u 1 \lambda_{y}} & e_{u 2 \lambda_{y}}
\end{array}\right)\left(\begin{array}{ll}
e_{u 1 x} & e_{u 2 x} \\
e_{u 1 y} & e_{u 2 y}
\end{array}\right)^{-1}\left(\begin{array}{l}
x \\
y
\end{array}\right) .
$$

The linear relation defining the UM is expressed in the form $\vec{\lambda}=M \vec{x}$, with the matrix $M$ defined as

$$
M=\left(\begin{array}{ll}
e_{u 1 \lambda_{x}} & e_{u 2 \lambda_{x}} \\
e_{u 1 \lambda_{y}} & e_{u 2 \lambda_{y}}
\end{array}\right)\left(\begin{array}{ll}
e_{u 1 x} & e_{u 2 x} \\
e_{u 1 y} & e_{u 2 y}
\end{array}\right)^{-1}
$$

Consider now the case when $x_{s}$ is an unstable focus. Here the eigenvalues $\alpha_{1,2}$ are real and $\left|\alpha_{1,2}\right|>1$. The point $\left(x_{s}, 0\right)$ is a saddle point of the extended map (17) with eigenvalues $\alpha_{1}, \alpha_{2}, \alpha_{1}^{-1}, \alpha_{2}^{-1}$ and corresponding eigenvectors $e_{u 1}, e_{u 2}, e_{s 1}$, and $e_{s 2}$. The eigenvectors corresponding to expanding motion have $\lambda$ components equal to zero, while the components for contracting eigenvectors are nonzero. The unstable manifold coincides with the coordinate plane, while the stable manifold is expressed by the relation $\vec{\lambda}=M \vec{x}$ with $M$ defined as

$$
M=\left(\begin{array}{ll}
e_{s 1 \lambda_{x}} & e_{s 2 \lambda_{x}} \\
e_{s 1 \lambda_{y}} & e_{s 2 \lambda_{y}}
\end{array}\right)\left(\begin{array}{ll}
e_{s 1 x} & e_{s 2 x} \\
e_{s 1 y} & e_{s 2 y}
\end{array}\right)^{-1}
$$

We can summarize the effect of noise as follows. In the case of a stable point, noise introduces in the system two unstable directions and creates an unstable manifold in the extended phase space. For an unstable point, the situation is precisely the opposite: noise induces stable directions and creates the stable manifold. The situation differs, however, in the case where $x_{s}$ is a saddle point of the noise-free system. Here, one of the eigenvalues, e.g., $\alpha_{1}$ will have a modulus bigger than one, whereas for the other eigenvalue the modu- lus is smaller. Stable and unstable directions are already present in the noise-free system. Noise then induces an additional stable direction and an additional unstable one. In the extended system, the unstable manifold is spanned by a "deterministic" eigenvector $e_{u 1}$ that has zero $\lambda$ components, and by $e_{u 2}$ having $\lambda$ components different than zero. In the same way, the stable manifold is spanned by a "deterministic" eigenvector $e_{s 1}$ and by a "fluctuational" eigenvector $e_{s 2}$. The matrix $M$ defining the unstable manifold of $x_{s}$ is given by

$$
M=\left(\begin{array}{ll}
0 & e_{u 2 \lambda_{x}} \\
0 & e_{u 2 \lambda_{y}}
\end{array}\right)\left(\begin{array}{ll}
e_{u 1 x} & e_{u 2 x} \\
e_{u 1 y} & e_{u 2 y}
\end{array}\right)^{-1}
$$

and in the same way the matrix defining the stable manifold is given by

$$
M=\left(\begin{array}{ll}
0 & e_{s 2 \lambda_{x}} \\
0 & e_{s 2 \lambda_{y}}
\end{array}\right)\left(\begin{array}{ll}
e_{s 1 x} & e_{s 2 x} \\
e_{s 1 y} & e_{s 2 y}
\end{array}\right)^{-1} .
$$

Consider now the case when the stationary point is a spiral node [46]. Its eigenvalues $\alpha_{1,2}$ are complex conjugates. If $\left|\alpha_{1,2}\right|<1, x_{s}$ is a stable spiral node, a generic vector on the unstable manifold is written as Eq. (18) with coefficients $c_{s 1,2}=0$, and the coefficients $c_{u 1,2}$ are arbitrary complex numbers. The matrix $M$ defining the unstable manifold is then calculated in the same way as in the case of a stable focus. It should be noted that, although the coefficients $\vec{e}_{u 1,2}$ are complex numbers, the final matrix $M$ is real. The stable manifold for $x_{s}$ is defined by the simple relation $\vec{\lambda}=0$.

In the case of an unstable spiral node, the stable and unstable manifolds swap their roles: The unstable manifold is defined as $\vec{\lambda}=0$ and the stable one by the matrix $M$.

Next, we investigate the case of a stable state of period $p$. It is an orbit defined by $\left\{x_{s 1}, x_{s 2}, \ldots, x_{s p-1}, x_{s p}\right\}$ with $x_{s p}=x_{s 1}$. A small neighborhood of $x_{s n}$ is mapped by Eq. (8) to a small neighborhood of $x_{s n+1}$ and the shape of the manifold can be calculated in the close vicinity of each of the points of the periodic orbit. We start by describing the manifold close to $x_{s 1}$. In a small neighborhood of $x_{s 1}$, the extended map can be written as

$$
\begin{gathered}
\delta x_{n+1}=\left[\left.\frac{\partial K}{\partial x}\right|_{x_{s 1}}\right] \delta x_{n}+\lambda_{n}=A \delta x_{n}+\lambda_{n} \\
\lambda_{n+1}=\left[\left.\frac{\partial K}{\partial x}\right|_{x_{s 2}}\right]^{-1 T} \lambda_{n}=B^{-1 T} \lambda_{n},
\end{gathered}
$$

where $\delta x_{n}$ is a small displacement about $x_{s 1}$ and $\delta x_{n+1}$ is a small displacement about $x_{s 2}$. The matrix $A$ is the Jacobian of the map calculated at $x_{s 1}$ and $B$ is the Jacobian matrix calculated at $x_{s 2}$. As in the case $p=1$, a generic point $x_{0}=(\delta x, \lambda)$ in a neighborhood of $x_{s 1}$ can be expanded as a combination of the eigenvectors of Eq. (26): $x_{0}=c_{s 1} e_{s 1}+c_{s 2} e_{s 2}+c_{u 1} e_{u 1}$ $+c_{u 2} e_{u 2}$ where the coefficients are real if the eigenvectors are real or complex if the eigenvectors are complex. As in the previous case of $p=1$, the unstable manifold of the orbit in the vicinity of $x_{s 1}$ is described by the matrix 


$$
M=\left(\begin{array}{ll}
e_{u 1 \lambda_{x}} & e_{u 2 \lambda_{x}} \\
e_{u 1 \lambda_{y}} & e_{u 2 \lambda_{y}}
\end{array}\right)\left(\begin{array}{ll}
e_{u 1 x} & e_{u 2 x} \\
e_{u 1 y} & e_{u 2 y}
\end{array}\right)^{-1},
$$

with the same notation as in Eq. (23). The same calculation can be repeated in order to calculate the local shape of the unstable manifold in the neighborhood of the other points forming the periodic orbit. The case $p=2$ will be considered explicitly below.

The case of a continuous flow, can be treated as usual as the limit for $h \rightarrow 0$ of the Euler integration scheme. Similarly a substitution of the Euler approximation $f\left(x_{n}\right)=x_{n}+h K\left(x_{n}\right)$ into Eq. (17) recovers a well-known result for the linearized Hamiltonian equations of continuous systems (cf., e.g., $[17,31])$

$$
\left\{\begin{array}{l}
\delta \dot{x}=K^{\prime}\left(x^{0}\right) \delta x+p, \\
\dot{p}=-K^{\prime}\left(x^{0}\right) p,
\end{array}\right.
$$

where $K^{\prime}\left(x^{0}\right)$ indicates the derivative of $K(x)$ calculated at $x^{0}$. In matrix form Eqs. (28) read as

$$
\left(\begin{array}{c}
\delta \dot{x} \\
\dot{p}
\end{array}\right)=\left(\begin{array}{cc}
B & 1 \\
0 & -B
\end{array}\right)\left(\begin{array}{c}
\delta x \\
p
\end{array}\right),
$$

where $B=K^{\prime}\left(x^{0}\right)$ and $\delta x$ is a small deviation of the coordinate from the fixed point attractor $x^{0}$. The generic point on the unstable manifold is written in this case as $x(t)$ $=c_{u 1} e_{u 1} \exp ^{\alpha_{1} t}+c_{u 2} v_{2}^{u} \exp ^{\alpha_{2} t}$ where $c_{u 1,2}$ are complex coefficients and $\alpha_{1}$ and $\alpha_{2}$ are two positive eigenvalues corresponding to the unstable eigenvectors $v_{1}^{\mu}$ and $v_{2}^{\mu}$. Accordingly, the connection between the momenta and coordinates on the unstable manifold of the linearized system can be written in a form similar to Eq. (21)

$$
\left(\begin{array}{l}
p_{x} \\
p_{y}
\end{array}\right)=\mathcal{M}\left(\begin{array}{l}
x \\
y
\end{array}\right),
$$

where

$$
\mathcal{M}=\left(\begin{array}{ll}
e_{u 1 p_{x}} & e_{u 2 p_{x}} \\
e_{u 1 p_{y}} & e_{u 2 p_{y}}
\end{array}\right)\left(\begin{array}{ll}
e_{u 1 x} & e_{u 2 x} \\
e_{u 1 y} & e_{u 2 y}
\end{array}\right)^{-1} .
$$

Equations (21) and (30) provide the choice of proper initial conditions for solution of the boundary values problem for flows and maps.

\section{LINEAR ANALYSIS OF THE ACTION SURFACE}

In the previous section, we addressed the problem of calculating the proper initial conditions to describe a trajectory performing the transition from a steady state to a remote final state. In order to calculate the transition probability, the nonequilibrium potential (or action) must be also known. In this section we perform a similar analysis in order to describe the action surface in the vicinity of the stationary points.

Consideration of the relation $\rho \propto \exp [-S / \epsilon]$ shows that the quasipotential exhibits local minima corresponding to the stable equilibrium orbits of the original system. Similarly, it displays local maxima corresponding to unstable orbits. For this reason, we expect to be able to express the potential in a
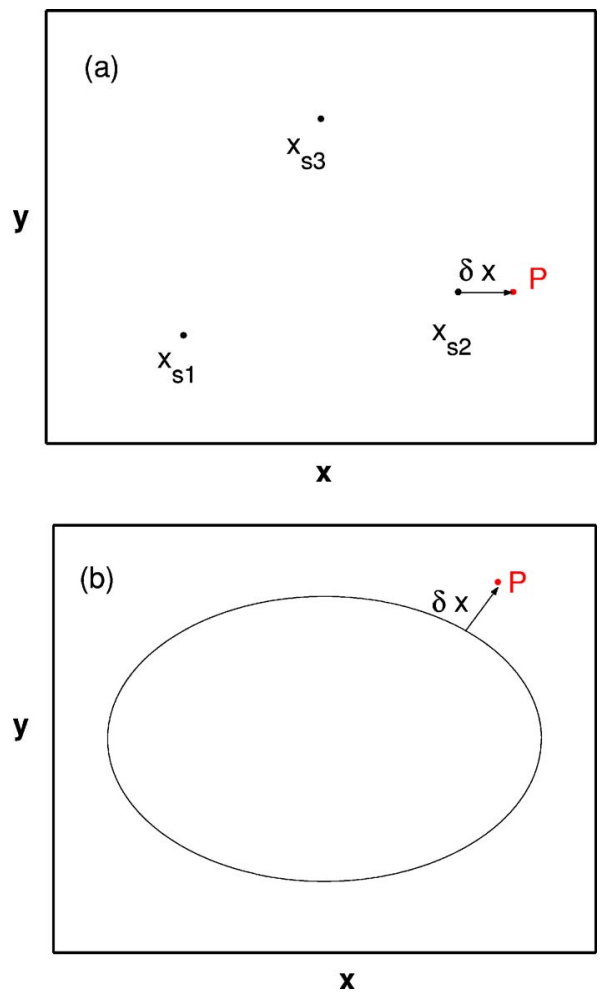

FIG. 2. (Color online) (a) Points in the vicinity of a period-3 orbit are described using the integer $i \in[1 \cdots 3]$ and a real vector $\delta x$ describing the displacement; (b) the region of coordinate space in the neighborhood of a limit cycle is described using a real parameter $\gamma$ and a vector $\delta x$ measuring the displacement.

neighborhood of stable equilibrium orbit as a quadratic form. For example, in the vicinity of a stationary point $x_{0}$, the potential can be approximated using a paraboloid

$$
S[\delta x]=S_{0}+\frac{1}{2} \delta x^{T} W \delta x
$$

where $S_{0}$ is the potential exactly on the stationary point and $\delta x$ represents a small deviation from $x_{0}$. The positively defined symmetrical matrix $W$ describes the shape of the potential around the stationary point. The case of a stable periodic structure is similar: For a periodic solution of period $p$ $\left\{x_{s 1}, x_{s 2}, \ldots, x_{s p-1}, x_{s p}\right\}$ with $x_{s p+1}=x_{s 1}$, the potential is still quadratic, but its shape differs in the vicinity of the different points of the orbit.

As shown in Fig. 2, a point in the vicinity of the stable orbit can be described by giving it an integer number indicating the closest point on the stable orbit, and a vector $\delta x$ giving the small displacement from that point. The potential can then be written as

$$
S[i, \delta x]=S_{0}+\frac{1}{2} \delta x^{T} W_{i} \delta x,
$$

where $i$ is the integer defining the point on the stable orbit and $W_{i}$ is the quadratic form defining the potential in the vicinity of $x_{s i}$.

The case of a periodic orbit in the continuous system is a limit for the discrete system. The space in the vicinity of a 
limit cycle can be described using a coordinate $\gamma$ along the cycle (in the same way as an integer was used in describing the space for a discrete system) and a vector $\delta x$ giving the small displacement from the cycle (as summarized in Fig. 2). The potential in this case is written as

$$
S[\gamma, \delta x]=S_{0}+\frac{1}{2} \delta x^{T} W(\gamma) \delta x,
$$

where $W(\gamma)$ is the quadratic form describing the potential.

It is clear from Eqs. (32)-(34) that a set of quadratic forms needs to be known in order to describe the boundary condition for the action. Similar to our calculation of the local shape of the Lagrangian manifold, we will start calculating $W$ for the simplest case of a period-one orbit, and we will then move toward more complicated stationary structures.

Consider the case of a period-1 stable point in a twodimensional map. In the vicinity of the stable point $x_{s}$, the dynamics of the map can be linearized to obtain Eq. (17)

$$
\begin{gathered}
\delta x_{n+1}=A \delta x_{n}+\lambda_{n}, \\
\lambda_{n+1}=A^{-1} \lambda_{n} .
\end{gathered}
$$

From the previous section, we know that the relation $\lambda$ $=M \delta x$ holds for the dynamics on the unstable manifold. Given a point $\delta x$ in the vicinity of the stable point, the corresponding action has to be calculated along the trajectory emanating from the steady state at $n \rightarrow-\infty$.

$$
S=\frac{1}{2} \sum_{-\infty}^{1} \lambda_{n}^{T} \lambda_{n}
$$

where the ${ }^{T}$ indicates the transpose operation on the matrix. Using the relation (21), the action can be rewritten as

$$
S=\frac{1}{2} \sum_{-\infty}^{1} \delta x_{n}^{T} M^{T} M \delta x_{n} .
$$

In order to express the action at the point $\delta x$, it is necessary to express the coordinate $\delta x_{n}$ as a function of $\delta x$. Manipulating the first equation in Eqs. (17), the motion of the coordinate $\delta x$ on the unstable manifold is expressed by $\delta x_{n+1}=(A$ $+M) \delta x_{n}$. In general we have $\delta x_{-n}=(A+M)^{-n} \delta x$. Substituting in the equation for $S$, the final expression for the action is

$$
S=\frac{1}{2} \sum_{1}^{\infty} \delta x^{T}(A+M)^{-2 n T} M^{T} M(A+M)^{-2 n} \delta x .
$$

The quadratic form $W=(A+M)^{-2 n T} M^{T} M(A+M)^{-2 n}$ is uniquely determined by the linearized system (17). Being a product of a matrix with its transpose, the eigenvalues of $W$ can be only positive or zero.

Consider now the case of a period-two stable orbit. In this case, the motion in the vicinity of the periodic orbit can be described using two different maps: one that maps points from a neighborhood of $x_{s 1}$ to a neighborhood of $x_{s 2}$, and one that maps from a neighborhood of $x_{s 2}$ to a neighborhood of $x_{s 1}$.

$$
\begin{array}{ll}
\delta x_{n+1}=A_{1} \delta x_{n}+\lambda_{n} & \delta x_{n+1}=A_{2} \delta x_{n}+\lambda_{n} \\
\lambda_{n+1}=A_{2}^{-T} \lambda_{n}
\end{array}, \begin{aligned}
& \lambda_{n+1}=A_{1}^{-T} \lambda_{n} \\
& A K / \partial x_{n}
\end{aligned}
$$

where $A_{1}=\partial K /\left.\partial x\right|_{x_{s 1}}$ and $A_{2}=\partial K /\left.\partial x\right|_{x_{s 2}}$. The first map describes the transitions from the vicinity of $x_{s 1}$ to the vicinity of $x_{s 2}$, the second map does the opposite.

The potential is a sum of different contributions: The increase in the potential due to jumps from the vicinity of $x_{s 1}$ to the vicinity of $x_{s 2}$, and the contribution due to the opposite type of jump. The potential in a neighborhood of $x_{s 1}$ is written as

$$
\begin{aligned}
S= & \frac{1}{2} \sum_{-\infty}^{0} \delta x_{0}^{T} M_{1}^{T} A_{1}\left(A_{2} A_{1}\right)^{n}\left(A_{2} A_{1}\right)^{T n} A_{1}^{T} M_{1} \delta x_{0} \\
& +\frac{1}{2} \sum_{-\infty}^{-1} \delta x_{0}^{T} M_{1}^{T}\left(A_{2} A_{1}\right)^{n}\left(A_{2} A_{1}\right)^{T n} M_{1} \delta x_{0}+S_{0} .
\end{aligned}
$$

Here the first sum counts the contributions due to jumps from $x_{s 2}$ to $x_{s 1}$, and the second sum contains the contribution of the jumps from $x_{s 1}$ to $x_{s 2}$. This gives the following expression for the quadratic form of the potential:

$$
\begin{aligned}
W_{1}= & M_{1}^{T}\left[\sum_{-\infty}^{0} A_{1}\left(A_{2} A_{1}\right)^{n}\left(A_{2} A_{1}\right)^{T n} A_{1}^{T}\right. \\
& \left.+\sum_{-\infty}^{-1}\left(A_{2} A_{1}\right)^{n}\left(A_{2} A_{1}\right)^{T n}\right] M_{1} .
\end{aligned}
$$

The expression for the potential in the vicinity of $x_{s 2}$ is obtained simply by exchanging the indices 1 and 2 in Eq. (41). The case of a generic period- $p$ orbit is a simple generalization of Eq. (41), where the contributions from $p$ different types of jump have to be considered.

Now consider the case of a continuous system. The potential can be calculated in the vicinity of stationary structures using either of two different approaches. The first consists of an application of the results obtained in the case of a map, using the limit $h \rightarrow 0$ in the Euler discretization scheme. In the case of a stable point, this gives

$$
S=S_{0}+\frac{1}{2} \delta x_{0}^{T} M^{T}\left(\int_{-\infty}^{0} e^{-A t} e^{-A^{T} t} d t\right) M \delta x_{0}
$$

and

$$
W=M^{T}\left(\int_{-\infty}^{0} e^{-A t} e^{-A^{T} t} d t\right) M,
$$

and, for a limit cycle, it gives

$$
W(\gamma)=M(\gamma)^{T}\left(\int_{-\infty}^{0} e^{-A t} e^{-A^{T} t} d t\right) M(\gamma),
$$

where the parameter $\gamma$ is the coordinate along the cycle and $\delta x$ is the displacement from the limit cycle. The local shape of the action calculated in the neighborhood of a limit cycle can be equivalently calculated by solving a Riccati equation with periodic boundary conditions [18]. It is worth noting that, in the case of a continuous system, the Hamiltonian 
formalism guarantees the well-known relation between the potential and the momenta

$$
p_{i}=\frac{\partial S}{\partial x_{i}}
$$

which implies $W=M$. However, this result cannot be exported straightforwardly to the case of discrete systems and, in the general case, $W \neq M$. An explicit example of this property will be given in the next section.

When an unstable state is considered, the situation can be different: The potential still exhibits a local maximum, but the simple quadratic description might not apply: The potential might display singular features and be wildly nondifferentiable $[17,21,31]$. Consider, for example, the IVDP system or the harmonically driven overdamped Duffing oscillator. In both systems a switching line converges asymptotically to the saddle cycle. As a result, a series of singularities in the action surface accumulate at the boundary and, given a small neighborhood of the cycle, the quasipotential there contains infinitely many nondifferentiable points.

\section{A. Example: 1D system}

To understand better the theory explained in the previous section, and in particular to show explicitly how the matrices $M$ and $W$ can differ for a generic map, we present as an example a simple one-dimensional linear system.

Consider the map $x_{i+1}=a x_{i}+\xi_{i}$, where $|a|<1$. The stochastic process $\xi$ has zero mean $\langle\xi\rangle=0$ and delta correlation $\left\langle\xi_{i} \xi_{j}\right\rangle=\epsilon \delta_{i j}$, where $\epsilon$ is the noise intensity. The corresponding extended map is

$$
\begin{gathered}
\delta x_{i+1}=a x_{i}+\lambda_{i}, \\
\lambda_{i+1}=\frac{1}{a} \lambda_{i} .
\end{gathered}
$$

The eigenvalues of the extended system are $a$ and $1 / a$. The stable manifold is defined by the relation $\lambda=0$ and the unstable manifold by the relation $\lambda=-(a-1 / a) x$; these give the matrix (in this case a scalar, in actuality) $M=-(a-1 / a)$. Motion on the unstable manifold is regulated by the equation $x_{i+1}=(1 / a) x_{i}$. The potential of a given point $x$ is calculated using

$$
S=\frac{1}{2} \sum_{-\infty}^{-1} \lambda_{i}^{2}=\frac{1}{2} \sum_{1}^{\infty}\left(a-\frac{1}{a}\right)^{2} a^{2 n} x^{2}
$$

and the summation is performed in the standard way to obtain $S=\frac{1}{2}\left(1-a^{2}\right) x^{2}$. Thus the matrix $W$ is found explicitly as $W=\left(1-a^{2}\right)$. So the matrices $W$ and $M$ are different.

In order to test these results, the system (46) was solved numerically by Monte Carlo simulation. First the stationary distribution was obtained by consideration of a collection of $10^{6}$ points (the system is obviously ergodic); the mean and the mean-square deviations were computed using the leastsquares technique, and their values compared with the theory as shown in Fig. 3. The agreement was found to be excellent for a wide range of $a$ and $\epsilon$ values.

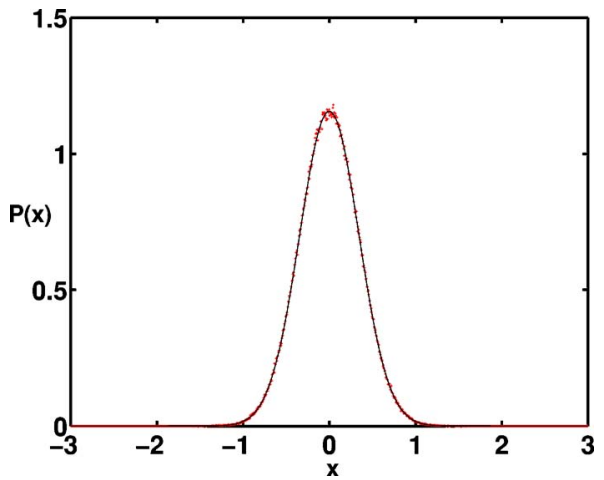

FIG. 3. (Color online) Comparison between numerical simulation (small dots) and theory (black thin line) for the system (46). The noise intensity is $\epsilon=0.1$, and parameter $a=0.4$. The numerical value of $W=0.842255$ and the theoretical value is $W=1-a^{2}$ $=0.840000$. The theory and the numerics almost completely overlap. Similar agreement has been obtained for a wide range of values of $a$ and noise intensities $\epsilon$.

As a second example, we consider a one-dimensional linear continuous system $\dot{x}=-a x+\xi$. The parameter $a$ is larger than zero and $\xi$ is a white uncorrelated Gaussian noise of intensity $\epsilon$. The extended Hamiltonian system is the limit as the integration step $h \rightarrow 0$ of the following map

$$
\begin{gathered}
\delta x_{i+1}=(1-a h) x_{i}+h \lambda_{i}, \\
\lambda_{i+1}=\frac{1}{1-a h} \lambda_{i} .
\end{gathered}
$$

Repeating the same calculation as above, the eigenspaces for map (48) are $\lambda=0$ and $\lambda=-1 / h[(1-a h)-1 /(1-a h)] x$. We then have $M=-1 / h[(1-a h)-1 /(1-a h)]$. The action for a given point is calculated using the summation procedure

$$
S=\frac{1}{2} h \sum_{-\infty}^{1} \lambda_{n}^{2}=\frac{h}{2} \sum_{1}^{\infty} \frac{1}{h^{2}}\left[(1-a h)-\frac{1}{1-a h}\right]^{2}(1-a h)^{2 n} x^{2} .
$$

Performing the summation we get

$$
S=\frac{1}{2 h}\left((1-a h)-\frac{1}{1-a h}\right)^{2} \frac{(1-a h)^{2}}{1-(1-a h)^{2}} x^{2},
$$

which gives $W=1 / h\left(1-(1-a h)^{2}\right)$. For a given finite value of $h$, the two matrices $W$ and $M$ are different. However, in the limit $h \rightarrow 0, M \rightarrow 2 a$, and $W \rightarrow 2 a$. This recovers the standard result $\lambda=\partial S / \partial x$ in the case of a continuous system.

\section{PARAMETRIZATION OF THE FAMILY OF TRAJECTORIES}

The first step toward the solution of the escape problem is the identification of a proper way of parametrizing the family of escape trajectories. Since Eq. (21) holds, it is enough to provide a parametrization of the trajectories in the coordinate space.

For a two-dimensional continuous system, in the vicinity of a stable point, the trajectories can be parametrized by 


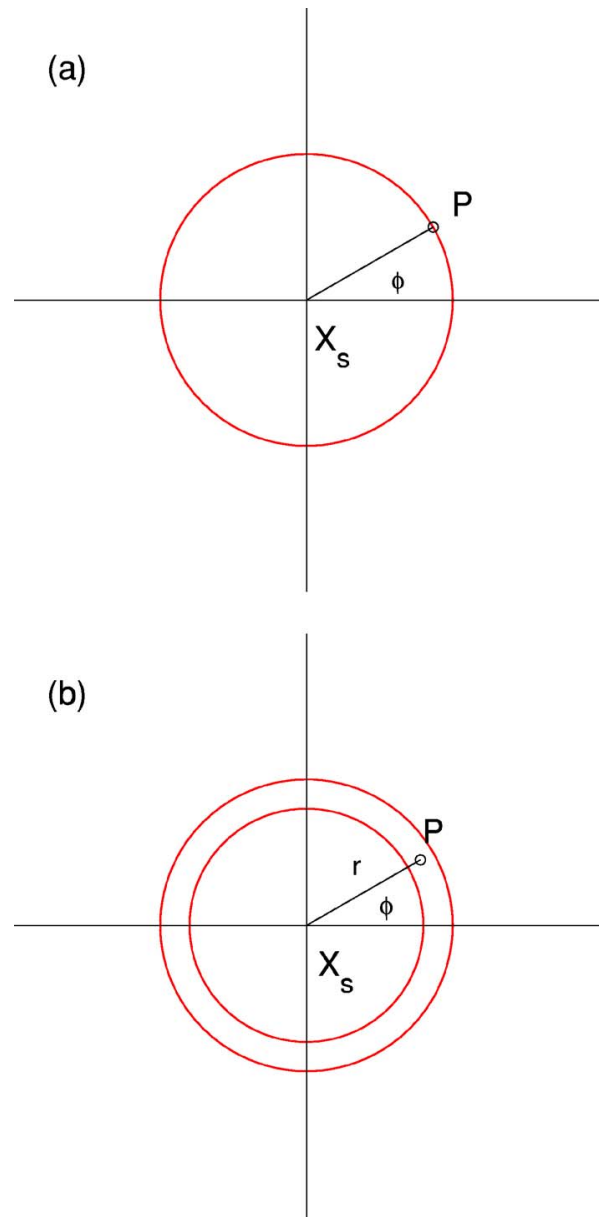

FIG. 4. (Color online) (a) Possible way to parametrize the unstable manifold of a stable point in a two-dimensional system. The point $P$ is chosen on a small circle (radius $\ll \sqrt{\epsilon}$ ); the angular position is the parameter we use to describe the set of escape trajectories on the manifold. (b) The parametrization of the trajectories for a two-dimensional map. The initial conditions are chosen inside an annulus limited by radii $r_{1}$ and $r_{2}$, whose values are chosen so as to cover all possible trajectories on the manifold.

choosing their initial conditions on a small circle $\left(S^{1}\right)$ centred on the point (see Fig. 4).

For a two-dimensional map, a larger space of parameters is required: it is diffeomorph [47] to a torus $T^{1}=S^{1} \times S^{1}$. The initial conditions can be chosen on a small annulus with extreme radii $r_{1}$ and $r_{2}$ as shown in Fig. 4. We are interested in giving a parametrization for all possible trajectories in the pattern. It is clear that this requires particular care in the choice of the possible radii $r_{1}$ and $r_{2}$. A wrong choice of $r_{1}$ and $r_{2}$ might result in some trajectories in the pattern not to be sampled with this choice. In order to include all the trajectories in the pattern the radii $r_{1}$ and $r_{2}$ must be chosen such that the iterations of the annulus cover the full manifold. Providing the radii to be small enough, it is sufficient to satisfy the condition for the linearized system around the stationary point. Once the extreme radii are chosen, a possible choice of parameters to describe the trajectories might be the radius $r$ of a small circle about the origin and the angular position $\phi$ on it (see Fig. 4).
In the general case, the parameter space for a $\mathrm{N}$-dimensional continuous system is diffeomorph to a $(\mathrm{N}$ -1)-dimensional product of tori $T^{n}$; for a $N$-dimensional map; it is diffeomorph to a $N$-dimensional product of tori $T^{n}$. Once initial conditions are defined on the coordinate space, the relation (21) is used to work out the corresponding momenta.

\section{ACTION PLOT AND THE SOLUTION OF THE BOUNDARY VALUE PROBLEM}

In this section we will show in a very effective way how the cost function can differ markedly for different escape trajectories and we will provide a tool for the solution of the boundary values problems.

To make it easier for the reader to follow the rationale of this section, we will stick to the case of a two-dimensional continuous system with a stable point and a saddle cycle; however, the discussion is actually more general and can be rewritten in an analogous way for the case of discrete systems. The theory described in the previous sections allows us to give a parametrization of the trajectories emanating from an initial stable structure (for the noise-free case) and spanning the unstable Lagrangian manifold. Each of these trajectories emanates from the stable state at $t \rightarrow-\infty$ and moves on the manifold in the $2 n$-dimensional phase space. In a projection they appear to wander around on the coordinate space and eventually they may escape the basin of attraction of the initial stable state. In order to give a good representation of the escape energy, we consider each trajectory in the pattern. As this path is on the manifold, it can be unambiguously described, providing parameters in the way described in Sec. IV. The cost of the trajectory (namely, its action) at the moment of escape is thus expressed unambiguously as a function defined on the parameter's space. We will refer to the graphical presentation of the action as a function of the parameters with the term action plot.

The behavior of the trajectories during the fluctuation allows us to identify two different regions in the parameter space. Trajectories in the first region can cross the boundary of the basin of attraction and move further to reach the basins of attraction of different stable points. A second class of trajectories has a different kind of dynamics: They first move toward the basin boundary, but their momentum is insufficient to allow them to cross the boundary; so they are reflected back to the interior of the basin of attraction. The two regions are separated by closed curves in the parameter space corresponding to heteroclinic trajectories. The values of the action corresponding to the two different regions are significantly different. This may be explained in the following way: The cost function $S$ evolves along the Hamiltonian trajectories according to Eq. (13). Thus $\dot{S}$ is a positively defined quadratic form in the auxiliary momentum $\vec{p}$. Consider now the most probable escape path $\gamma_{0}$. During the fluctuation from the initial state toward the boundary, $\vec{p}$ is significantly different than zero and the cost function increases along the motion. As the system approaches the cycle, $\vec{p}$ decreases exponentially and the optimal path converges to the limit cycle asymptotically. At the same time the cost function ap- 


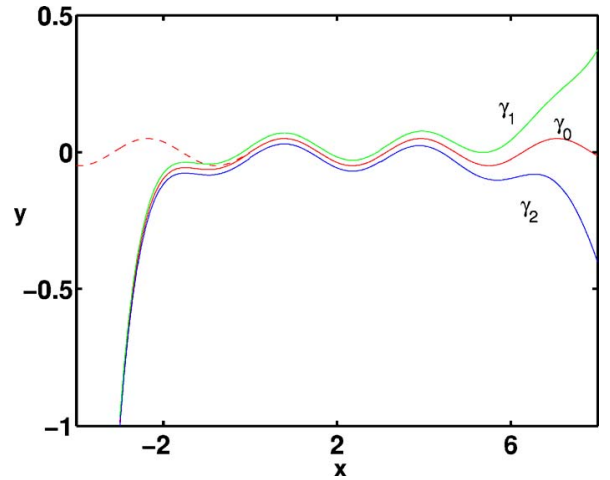

FIG. 5. (Color online) Three different classes of trajectories are possible for a system with a saddle cycle. $\gamma_{0}$ is the heteroclinic path: It reaches the cycle (dashed line) asymptotically for $t \rightarrow+\infty$; $\gamma_{1}$ has a bigger momentum and crosses the cycle; $\gamma_{2}$ has insufficient momentum to reach the cycle and it is reflected back.

proaches its asymptotic value $S_{0}$ corresponding to the activation energy. Consider a path $\gamma_{1}$ that is a perturbation of the optimal path. As a first possibility, the momentum $|\vec{p}|$ on $\gamma_{1}$ may be bigger than on $\gamma_{0}$; as a result, $\gamma_{1}$ hits the cycle with a residual momentum. The cost function calculated along $\gamma_{1}$ $S_{1}=\int_{\gamma_{1}}|\vec{p}|^{2} d t$ will be slightly higher than $S_{0}$. Changing the trajectory continuously, it may have a cost $S_{1}$ indefinitely close to $S_{0}$.

A second class of trajectories is also possible: Consider a trajectory $\gamma_{2}$ having a slightly smaller momentum than $\gamma_{0}$. Such a trajectory cannot overcome the repelling force of the limit cycle: It will come very close to the the cycle, but it will be repelled back toward the initial state. In this process, its cost $S_{2}$ remains slightly smaller than the activation energy $S_{0}$. During the motion toward the stable state, the momentum of $\gamma_{2}$ stays very small and its cost will not increase significantly. After reaching some internal part of the basin of attraction, the fluctuational motion will start once more and then the path will again move toward the limit cycle. During this second outward motion, its momentum becomes once more significantly different than zero. For this reason, when $\gamma_{2}$ away from the limit cycle, its cost will be significantly bigger than the activation $S_{0}$. For this second class of trajectories, it is impossible to approach the cost $S_{0}$ by changing the momentum continuously: A discontinuity in the cost function is always present. The different behaviors of the trajectories are summarized in Fig. 5.

According to the previous discussion, the cost function is a discontinuous function in the parameter space and the discontinuities correspond to heteroclinic trajectories. The lowest-cost heteroclinic trajectory is the optimal path.

\section{A. Self similarity of the action plot}

The structure of the action plot for a generic nonequilibrium system with a limit cycle can be very complicated. In Fig. 6(a) the action plot is shown for the inverted van der Pol oscillator (which will be discussed in more detail Sec. VII B). The features that will be described for this system are in fact very general, as will become apparent from the discussion.
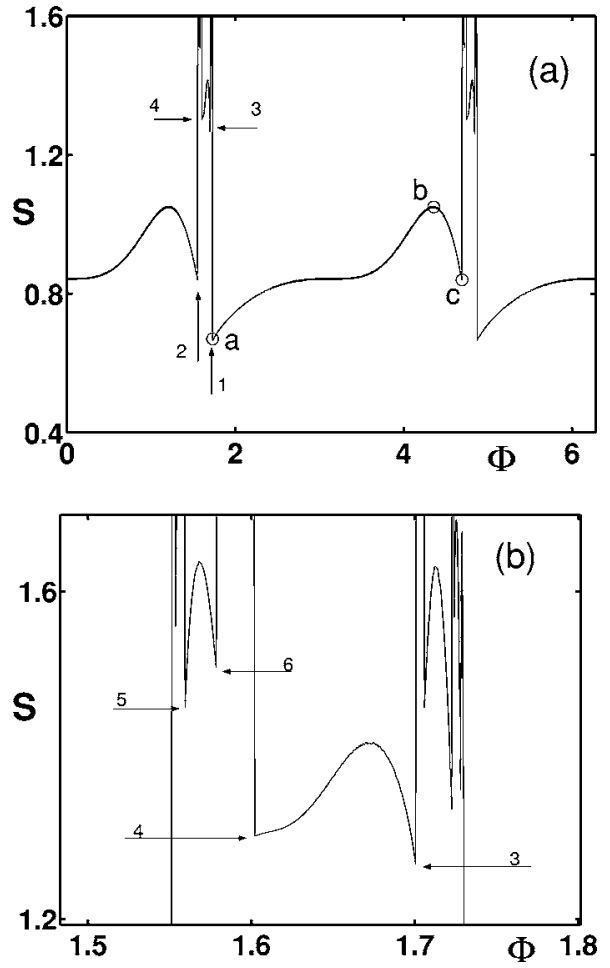

FIG. 6. (a) The action plot for the IVDP system. The typical hills-peaks structure is evident. Arrows (1) and (2) show two discontinuity points in the first hill. Arrows (3) and (4) show other discontinuities in the second hill. (b) A zoom of the first peak [area included between the points marked by the arrows (1) and (2)] reveals the self-repeating structure of the action plot. The points marked with (5) and (6) are other discontinuity points in the peak.

It is evident that the action plot has a wildly singular structure composed of broad, rounded, "hills" [for example, the region a-c in Fig. 6(a)] and very sharp "peaks." At the edges of the hills, discontinuities are present, such as those marked by (1) and (2) in Fig. 6(a). According to the discussion above, each of the discontinuities corresponds to a heteroclinic trajectory. The discontinuity with the lowest action [marked as (1)] corresponds to the optimal path. On the other hand, the presence of other discontinuities suggests the presence of other nonoptimal heteroclinic paths.

A zoom inside the first peak [Fig. 6(b)] reveals the presence of closely similar structure: A smooth hill with two sharp peaks at each side. The discontinuities between hill and peaks are marked by arrows (3) and (4). Inside the peaks, more hills and peaks are present. Two more discontinuities are marked with arrows (5) and (6). Such a structure continues indefinitely: Smaller and smaller hills exist, having higher and higher cost, and infinitely many discontinuities are present in the action plot.

The complex structure described here can be understood on the basis of the arguments introduced in Sec. VII. The first hills correspond to trajectories that cross the cycle during their fluctuation. The trajectories in the peaks have a significantly different cost: They are paths that have been reflected back and experienced the (cost-demanding) fluctuational motion twice. Inside the peaks, trajectories that are reflected back only once form the lower hills and trajectories 
that are reflected back more than once form the internal peaks. This process goes on indefinitely and a hierarchy of trajectories can be built according to the number of reflections back from the limit cycle that they experience.

The peculiar structure of the action plot can be used to locate the heteroclinic trajectories in the system and, in particular, the optimal path. The optimal path corresponds to the minimum action heteroclinic path; other heteroclinic trajectories present in the system are nonoptimal.

\section{B. IVDP system}

As our first application, we study the activation energy for noise-induced escape in the inverted van der Pol oscillator [48]. The IVDP has already been mentioned above: It is a nonlinear oscillator containing terms describing losses and energy pumping. The dynamical model is

$$
\ddot{x}=-2 \eta\left(1-x^{2}\right) \dot{x}-x+\sqrt{4 \eta T} \xi(t),
$$

where $x$ is a dynamical variable and $\xi(t)$ is a noise process. In what follows we will assume $\xi(t)$ to be a zero-mean white Gaussian process. Its correlation function is $\langle\xi(t) \xi(s)\rangle=\delta(t$ $-s)$. The intensity of the noise term is $\epsilon=\sqrt{4 \eta T}$ where $T$ is the thermodynamic temperature of the system. The positive parameter $\eta$ in Eq. (51) regulates not only the friction but also the pumping of energy into the system, which keeps the system away from thermodynamic equilibrium. As a result, the activation energy of this system displays a dependence on the value of $\eta$ that is nontrivial.

The dynamics of the system can be better understood with the substitution $y=\dot{x}$. In this way Eq. (51) can be rewritten as

$$
\begin{gathered}
\dot{x}=y, \\
\dot{y}=-2 \eta\left(1-x^{2}\right) y-x+\sqrt{4 \eta T} \xi(t) .
\end{gathered}
$$

In the coordinate space $x-y$, the noise-free dynamics of the system can be summarized as follows: The origin $O=(0 ; 0)$ is a stable equilibrium point. Its basin of attraction $\Omega$ is limited by a saddle cycle. The stability of $O$ depends on the parameter $\eta$ and can be studied using linear analysis. In the vicinity of $O$, the system becomes

$$
\left(\begin{array}{l}
\dot{x} \\
\dot{y}
\end{array}\right)=\left(\begin{array}{cc}
0 & 1 \\
-1 & -2 \eta
\end{array}\right)\left(\begin{array}{l}
x \\
y
\end{array}\right) \text {. }
$$

The nature of the fixed point depends on the eigenvalues of the system $\lambda_{1,2}=-\eta \pm \sqrt{\eta^{2}-1}$. For values of $\eta$ smaller than 1 , $\lambda_{1,2}$ are complex conjugates and the fixed point is a spiral node; for $\eta=1$ the system bifurcates, $\lambda_{1,2}$ become real and $O$ becomes a stable node. The particular case $\eta=0$ correspond to an ideal harmonic oscillator of frequency 1 .

When noise is added in the system, transitions from the stable origin to the limit cycle become possible. As discussed above, when the noise intensity is small, noise-induced escape from the basin of attraction of the fixed point is governed by the properties of the most probable escape path, which is the heteroclinic trajectory of lowest cost. As the noise intensity $\epsilon$ depends on both temperature and friction

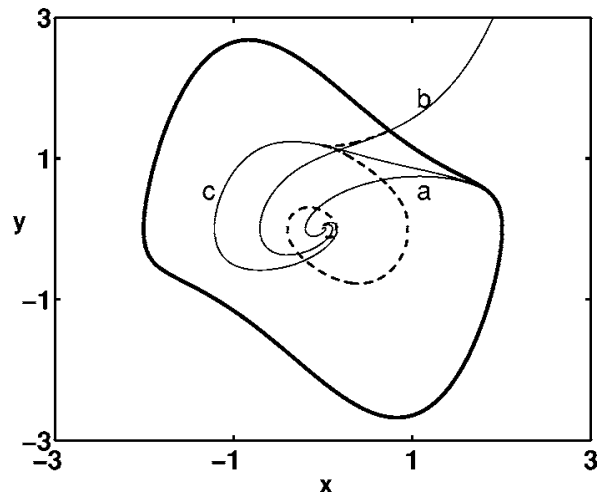

FIG. 7. Interesting paths (solid lines) in the pattern of optimal trajectories and caustics (dashed line) for the IVDP system. A caustic present in the system. "a" is the optimal escape path: It is the heteroclinic trajectory with the least action. " $b$ " is the trajectory that hits the cycle in the same point of the external branch of the caustic. "c" is a nonoptimal heteroclinic path.

coefficient, the parameter that becomes indefinitely small is $T$ while the parameter $\eta$ is held at a fixed value.

The action plot can be used successfully to calculate the activation energy in the system. As discussed in Sec. V, the pattern of trajectories is parametrized by setting the initial conditions on a small circle around $O$. The parameter space is thus diffeomorph to a $S^{1}$. The action plot as a function of the angular position $\phi$ on the circle is shown in Figs. 6(a) and 6(b). Interesting paths in the pattern of optimal trajectories are shown in Fig. 7 together with singularities in the pattern (dashed lines).

The optimal escape path is marked with the letter "a." It reaches the cycle tangentially for $t \rightarrow \infty$. It corresponds to the discontinuity "a" in Fig. 6(a). Its action corresponds to the absolute minimum in the action plot. The path "b" in Fig. 7 corresponds to the top of the first hill in Fig. 6(a). Its cost is the maximum cost possible for a trajectory in the first hill. The path "c" in Fig. 7 is a nonoptimal heteroclinic path: It reaches the cycle asymptotically for $t \rightarrow \infty$, but its cost is bigger than the activation energy (the cost of path "a"). It corresponds to the discontinuity "c" in Fig. 6(a). It is possible to move continuously from path "a" to path "c" along the first hill by continuously changing the parameter $\phi$ in the action plot.

Due to the central symmetry in the system of Eqs. (52) two trajectories, such that one is the central reflection of the other, have the same cost. Due to this symmetry, the action plot, as a function of $\phi$, has a period of $\pi$ and not $2 \pi$. This means that two degenerate optimal paths are present in the system: the path "a" in Fig. 7 and that obtained from "a" by central reflection. In Fig. 7 the singularities in the pattern of optimal trajectories are shown as dashed lines. They are caustics, i.e., envelopes of Hamiltonian trajectories. It appears clearly evident that a cusp point separates the caustic into two branches: an external branch that hits the limit cycle, and an internal one that moves toward the origin. The trajectory "b" hits the limit cycle and simultaneously touches the caustic.

In Fig. 8, the dependence of $S$ on $\eta$ (full curve) calculated using the action-plot technique is compared with Monte 


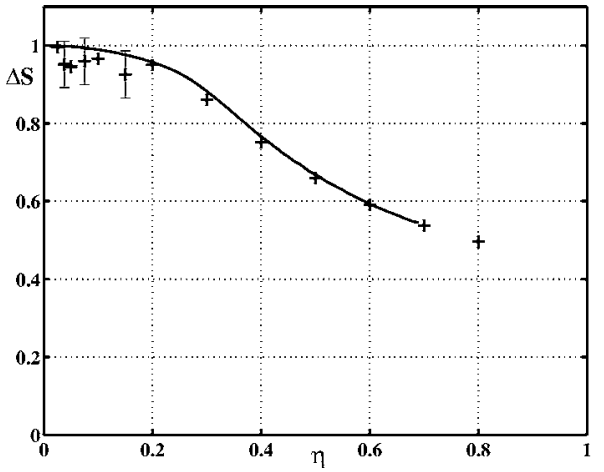

FIG. 8. The activation energy for escape in the inverted van der Pol oscillator as a function of the $\eta$ parameter. The results of Monte Carlo simulations (crosses) are compared with the action calculated using the action plot technique (full curve). Error bars are shown for some of the points.

Carlo simulations (crosses) in the limit of small noise intensity. Theoretical predictions and the results of the simulations agree well over a wide range of parameter values. The small$\eta$ asymptotic behavior of the activation energy corresponds to theoretical predictions based on adiabatic elimination of the fast rotation. The value $\eta=0$, corresponding to deterministic motion of the system, may be reached only as a limit.

\section{Harmonically driven overdamped Duffing oscillator}

The inverted van der Pol oscillator described in Sec. VII B represents an archetypal nonequilibrium autonomous system with a limit cycle. In this section we show how the same method can be applied to the case of a nonautonomous system with limit cycles. In particular, we consider a potential system kept out of equilibrium by a time dependent external force. Consider a bistable potential $U=-\frac{1}{2} x^{2}+\frac{1}{4} x^{4}$ (Duffing potential) and consider a particle moving according to

$$
\dot{x}=-\nabla U+A \sin (\omega t)+\sqrt{\epsilon} \xi(t) .
$$

Here the harmonic driving keeps the system away from equilibrium so that the detailed balance condition does not hold; the amplitude $A$ of the driving force is chosen large enough to be beyond the perturbative regime. The driving frequency is comparable with other time scales present in the system. The noise is introduced in the model by the stochastic variable $\xi$. In what follows, we consider $\xi$ to be a standard zeromean white Gaussian noise. Its correlation function is $\langle\xi(t) \xi(s)\rangle=\delta(t-s)$. The one-dimensional system (54) is a nonautonomous; in order to make it an autonomous system, the following auxiliary system is introduced:

$$
\begin{gathered}
\dot{x}_{1}=\omega, \\
\dot{x}_{2}=-\nabla U+A \sin \left(x_{1}\right)+\sqrt{\epsilon} \xi(t) .
\end{gathered}
$$

The system (55) is two-dimensional, but it is now autonomous.

The presence of the external driving transforms the equilibrium points to limit cycles of period $2 \pi / \omega$ : The minima of

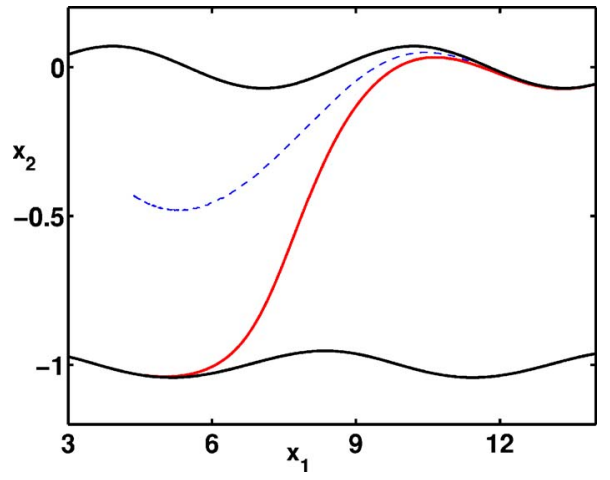

FIG. 9. (Color online) The optimal escape trajectory for the overdamped Duffing oscillator. Here the frequency of the harmonic driving is set to $\omega=1$ and the amplitude to $A=0.1$. The optimal path is shown by a full line. It is clearly a heteroclinic trajectory. The dashed line is the switching line for the system. It is clear from the figure that the switching line and MPEP never cross, but they asymptotically touch when they converge to the cycle as $t \rightarrow \infty$.

the potential $U$ for $x= \pm 1$ become stable limit cycles and the maximum of the potential becomes a saddle cycle. The presence of noise in the system might induce transitions from one stable cycle to the other one, across the unstable cycle. In order to calculate their probability, the extended system

$$
\begin{gathered}
\dot{x}_{1}=\omega, \\
\dot{x}_{2}=-\nabla U+A \sin \left(x_{1}\right)+p_{2}, \\
\dot{p}_{1}=-A \cos \left(x_{1}\right) p_{2}, \\
\dot{p}_{2}=\frac{d^{2} U}{d x_{2}^{2}} p_{2},
\end{gathered}
$$

has to be solved with a proper choice of boundary conditions: $\left(x_{1} ; x_{2}\right)$ must be on the stable cycle for $t \rightarrow-\infty$ and on the saddle cycle for $t \rightarrow \infty$.

The trajectories emanating from the stable cycle are a one-parameter family on the unstable manifold. A possible choice of parametrization can be as follows. Let the initial conditions be chosen to be of form $\left(x_{1} ; x_{2}\right)=\left(x_{1 c}(\phi), x_{2 c}(\phi)\right.$ $+\delta$ ), where $x_{1 c}$ and $x_{2 c}$ are the coordinates of the stable cycle at a given phase $\phi$ and $\delta$ is a small displacement in the $x_{2}$ direction. The parameter $\phi$ parametrizes the family of trajectories. The optimal path found for the system is shown in Fig. 9. It is an heteroclinic trajectory reaching the saddle cycle for $t \rightarrow \infty$.

\section{Henon map}

As a second example of the application of the formalism, we consider the case of a noise-driven Henon map. The Henon map, introduced in 1976 by Henon [49], takes the form 


$$
\left\{\begin{array}{l}
x_{n+1}=a-x_{n}^{2}+b y_{n}+\xi_{n} \\
y_{n+1}=x_{n} .
\end{array}\right.
$$

Here $x_{n}$ and $y_{n}$ are the dynamical variables; $a$ and $b$ are real parameters for the system and $\left\{\xi_{n}\right\}$ is a set of uncorrelated white Gaussian noises of intensity $\epsilon$. Different regimes of complexity can be obtained in this system by tuning the parameters in an appropriate way (see, for instance, $[49,50])$. In this section we will apply the technique described to regimes which are more and more complex. We start with a choice of parameters $(a=1.1, b=-0.3)$ corresponding to one stable period-1 orbit and one attractor at infinity. The boundary of the basin of attraction is then smooth (nonfractal) and a period-1 saddle orbit is present in the boundary. The extended system corresponding to Eq. (57) is

$$
\left\{\begin{array}{l}
x_{n+1}=a-x_{n}^{2}+b y_{n}+\lambda_{x n}, \\
y_{n+1}=x_{n}, \\
\lambda_{x n+1}=\lambda_{y n}, \\
\lambda_{y n+1}=\frac{\lambda_{x n}+2 x_{n+1} \lambda_{x n}}{b}
\end{array}\right.
$$

and the activation energy evolves along the trajectory according to $E_{n+1}=E_{n}+\frac{1}{2} \lambda_{x n}^{2}$.

In the extended system (58), the stable orbit becomes a saddle, and the escape trajectories span its unstable manifold. According to the general theory, it is a two-dimensional surface and the trajectories form a two-parameter family. From the linearization of the system (58), the manifold can be defined in a neighborhood of the initial state and a linear relation (21) between coordinates and momenta is obtained. The matrix $M_{i j}$, which appears in Eq. (21), is here a $2 \times 2$ matrix.

A suitable choice for the parametrization of the manifold about the initial state is the radius and the angular position on a small circle around the point. The action plot is here a function $S^{1} \times S^{1} \rightarrow$ R. The situation is very complicated, but a global minimization is still possible and an "energy minimal" escape trajectory can be found, as shown in Fig. 10. The theoretical path obtained using the actionplot techniques is shown as a dashed line. It is compared with the results of a Monte Carlo simulation using a noise intensity $D=0.02$. The two paths are in almost perfect agreement. The optimal trajectory leaves the initial state along its unstable manifold, reaches the boundary, and then drifts in an almost noise-free way toward the saddle at $(-1.88 ;-1.88)$. The escape takes place at the saddle point.

In order to show that the method described is general, we tune the parameters to a particularly interesting regime where the basin boundaries are fractal. Considering $a=1.405$ and $b=-0.3$, the system displays a stable period- 2 orbit $\left(x_{s 1} ; x_{s 2}\right)$ coexisting with a stable attractor at infinity. The boundary of the basin of attraction is fractal and a period-3 accessible orbit $[37,51]$ is embedded in it. It is an example of locally disconnected fractal boundary (see the discussion in [52] for the classification of fractal boundaries). The correct mathematical definition of this property is the following:

Definition 1 (locally disconnected set). A set $A$ is defined

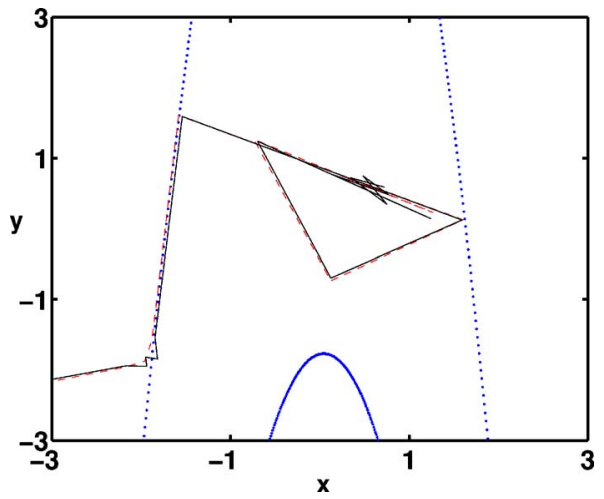

FIG. 10. (Color online) Comparison of theory and numerical simulations for the escape problem in the Henon map (parameters are $a=1.1$ and $b=-0.3$ ). The dots indicate the basin boundary of the stable orbit (the boundary is, of course, continuous; the dots appear due to finite resolution). The theoretical MPEP (dashed line) and numerical (full line) escape paths are in excellent agreement. Escape takes place through the saddle point in $(-1.88 ;-1.88)$

as locally disconnected if, given a point $x$ in the set, there exists a small ball of radius $\epsilon$, such that for every $\delta$ a point $y \in A$ exists for which $|x-y| \leqslant \delta$ and no connected subset of $A$ containing both $x$ and $y$ and lying wholly in the $\epsilon$-ball can be found.

The extended system (58) must be solved with the correct initial conditions in a small neighborhood of the period-two stable orbit. The trajectories solution of Eq. (58) span a twoparameter family on the two-dimensional unstable manifold. In order to provide appropriate initial conditions, the full extended system (58) is linearized in the vicinity of one of the points in the stable orbit, for instance $x_{s 1}$. The expanding subspace was calculated using linear analysis, and the matrix relating coordinates and momenta on the manifold were worked out.

Although the action plot for the system is again very complicated, global minimization can be performed to obtain the MPEP. The results of the theory agree very well with the numerical output of Monte Carlo simulation as shown in Figs. 11 and 12. The escape trajectory leaves the period-two stable cycle along the optimal path and reaches the boundary through the stable manifold of the accessible period-three orbit. Investigation of the almost noise-free dynamics along the fractal boundary lies beyond the scope of the present work but has been discussed in general terms elsewhere [37].

In this section, we have demonstrated the method explicitly for the Henon map, first for a parameter choice corresponding to a stable period-one orbit and smooth basin boundaries and, second, for the more complex case of a period-two stable orbit and fractal basin boundaries. It is clear that the method can be applied in the general case of a period- $n$ initial orbit: The two-parameter characterization of trajectories still holds, and the procedure described above also applies to the case of a period- $n$ initial state.

\section{E. Julia map}

With the following example, we aim to revise the case of activation processes in systems where the fractal basin 


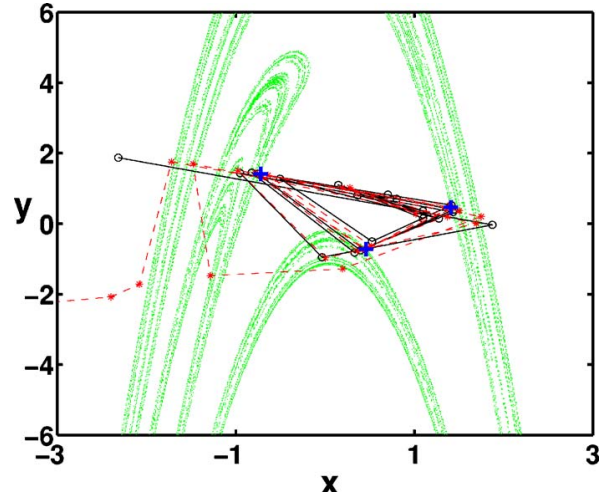

FIG. 11. (Color online) Comparison of theory and experiment for the escape problem in the Henon map (parameters are $a$ $=1.405$ and $b=-0.3$ ). The solid line is a numerical realization of the escape; the dashed line is the theoretical trajectory found using the actionplot. Agreement between them inside the domain of attraction is excellent. On the boundary, the numerical dynamics diverges from theory due to the finiteness of the noise.

boundaries are locally connected (LC) [52]. The mathematical definition of a locally connected basin boundary is as follows:

Definition 2 (locally connected set). A set $A$ is defined connected if, given a point $x$ in the set and a small ball of radius $\epsilon$, around it, a $\delta$ such that for every $y \in A$ with $\mid x$ $-y \mid \leqslant \delta$ there is a connected subset of $A$ containing both $x$ and $y$ and lying wholly in the $\epsilon$-ball.

An example of this kind of fractal boundary is given by the following analytic map $[52,51]$

$$
\begin{gathered}
x_{n+1}=x_{n}^{2}-y_{n}^{2}+a x_{n}+\xi_{x n}, \\
y_{n+1}=2 x_{n} y_{n}+a x_{n}+b y_{n}+\xi_{y n} .
\end{gathered}
$$

Here $x_{n}$ and $y_{n}$ are two dynamical variables and $a$ and $b$ are real parameters. The two stochastic terms $\xi_{x n}$ and $\xi_{y n}$ are Gaussian and $\delta$ correlated with intensity $\epsilon$. Choosing the

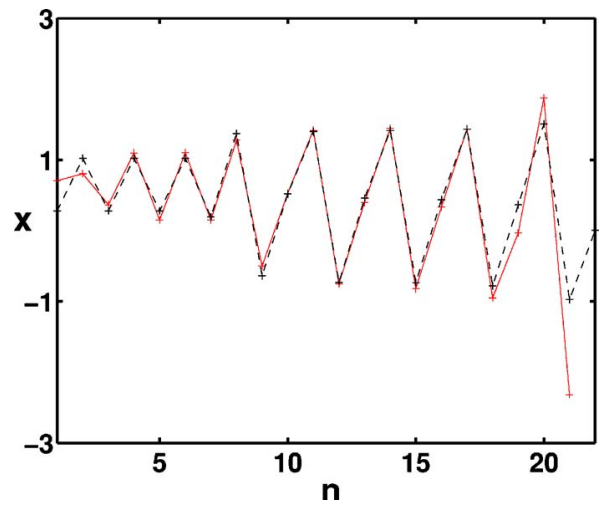

FIG. 12. (Color online) The theoretical MPEP (dashed line) for the Henon map compared with an escape path (solid line) found by numerical simulation in the iteration-coordinate domain: $n$ represents the number of iterations (the origin $n=0$ is chosen arbitrarily) and $x$ is the coordinate. The parameters are $a=1.405$ and $b=-0.3$. Agreement between experiment and theory is excellent.

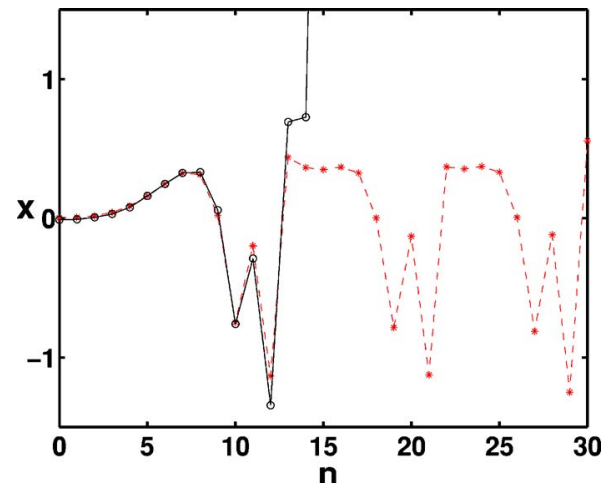

FIG. 13. (Color online) Comparison of theory and numerical simulations for the escape problem in the Julia map. The theoretical MPEP (dashed line) and numerical (full line) escape paths agree well inside the basin of attraction. Escape takes place through the saddle cycle of period 9 .

parameter $a=0.7$ and $b=0.5$ (a choice made according to [50]), the system has a stable fixed point at $(x, y)=(0,0)$ whose basin boundaries are fractal and locally connected. In particular, the basin boundaries are a Julia set [53], historically an example of fractals that were investigated by the French mathematician Gaston Julia (1893-1978).

The presence of the two noise sources $\xi_{x n}$ and $\xi_{y n}$ makes it possible to observe transitions from the stable point of Eq. (59) to the basin boundary. Previous investigation of the activation process in this system was reported by Grassberger. His analysis was difficult due to the fractal structure of the boundaries; in particular, he failed to locate the correct boundary conditions.

Using the asymptotic approach in the limit of $\epsilon \rightarrow 0$, the extended map is obtained

$$
\begin{gathered}
x_{n+1}=x_{n}^{2}-y_{n}^{2}+a x_{n}+\lambda_{x n}, \\
y_{n+1}=2 x_{n} y_{n}+a x_{n}+b y_{n}+\lambda_{y n}, \\
\lambda_{x n+1}=\frac{\left(2 x_{n+1}+b\right) \lambda_{x n}}{J}-\frac{\left(2 y_{n+1}+a\right) \lambda_{y n}}{J}, \\
\lambda_{y n+1}=\frac{2 y_{n+1} \lambda_{x n}}{J}+\frac{\left(2 x_{n+1}+a\right) \lambda_{y n}}{J} .
\end{gathered}
$$

The trajectories solution of Eq. (60) forms a two-parameter set in the extended four-dimensional space. The space of parameters is once more diffeomorph to a torus $T^{2}$. The trajectory that minimizes the cost is shown together with the results of numerical simulations in Fig. 13. The optimal escape path agrees with the results of the numerical simulations. It reaches the boundary asymptotically and the escape takes place following a saddle cycle of period 9 .

In order to compare more accurately the theoretical MPEP with the results of numerical simulations, time series of the $x$ variable for both the theoretical MPEP and the numerical one are shown together in Fig. 14. Excellent agreement is evident. The theoretical MPEP escapes through the period 9 orbit. but the numerical MPEP escapes earlier due to finite 


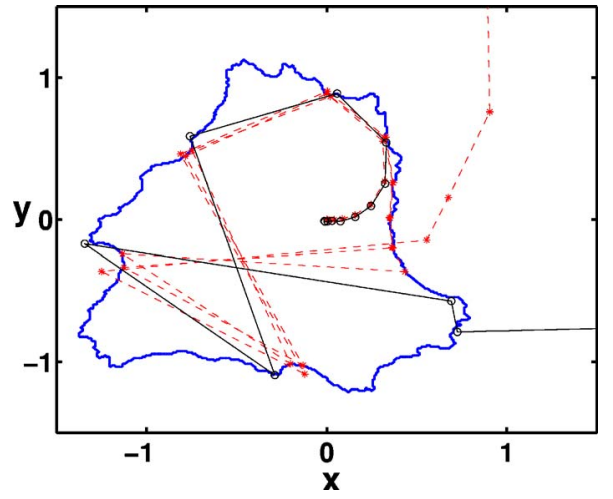

FIG. 14. (Color online) Comparison of theory and numerical experiment for the escape problem in the Julia map in the coordinate space $x-y$. The theoretical MPEP (dashed line) and numerical (full line) escape paths agree well. The dynamics of the MPEP on the boundary follows a saddle cycle of period 9 .

noise diffusion. We still do not know why the boundary conditions are such.

\section{CONCLUSION}

In this paper we have carried out a wide-ranging investigation of activation processes in complex nonlinear systems and maps. The problem has been addressed in the asymptotic regime of small noise intensity. In this limit, the features of activation have been investigated using an extended areapreserving map for the discrete systems and an auxiliary Hamiltonian system for continuous flows.

In particular, we focused on the solution of boundary value problems which appear in the context of activation through a saddle. Difficulties arising in the solution of these problems due to the wild nondifferentiable structure of the unstable manifold have been reviewed and tackled. All possible escape trajectories have been parametrized using a minimal number of parameters and the activation cost has been calculated as a function of these parameters. Minimization has been performed to calculate the MPEP and the activation energy. A graphical presentation of this result was dubbed the action plot.

It is clear from the structure of the action plot that infinitely many heteroclinic trajectories lie in a narrow region of the parameter space. This makes it extremely hard to isolate the optimal path among all other heteroclinic trajectories using only information on the momenta. The action plot provides the information required: Besides locating (at least in principle) all heteroclinic trajectories in the pattern, it sorts them in a hierarchy and makes it easier to identify the MPEP among them. We have shown several examples of qualitatively different systems that can be successfully treated using the action-plot technique, starting from the inverted van der Pol system as an archetype of an autonomous system with a limit cycle, to the periodically driven overdamped Duffing oscillator. In order to show the method for maps, the Henon map has been investigated in different ranges of parameters, corresponding to smooth boundaries and locally disconnected fractal boundaries. A quadratic Julia map displaying locally connected fractal boundaries has been also investigated. In all these cases, the theoretical prediction agrees with the numerical results over a broad range of parameters and through several bifurcations.

\section{ACKNOWLEDGMENTS}

We are grateful for valuable discussions with Igor Khovanov and Suso Kraut. The research has been supported in part by the Engineering and Physical Sciences Research Council (U.K.), INTAS, and the Russian Foundation for Fundamental Sciences (RFFI).
[1] M. I. Dykman, D. G. Luchinsky, R. Mannella, P. V. E. McClintock, N. D. Stein, and N. G. Stocks, Nuovo Cimento D 17, 661 (1995).

[2] F. Jülicher, F. Ajdari, and J. Prost, Rev. Mod. Phys. 69, 1269 (1997).

[3] M. O. Magnasco, Phys. Rev. Lett. 71, 1477 (1993).

[4] M. I. Dykman, H. Rabitz, V. N. Smelyanskiy, and B. E. Vugmeister, Phys. Rev. Lett. 79, 1178 (1997).

[5] V. N. Smelyanskiy, M. I. Dykman, H. Rabitz, B. E. Vugmeister, S. L. Bernasek, and A. B. Bocarsly, J. Chem. Phys. 110, 11488 (1999).

[6] M. B. Willemsen, M. P. van Exter, and J. P. Woerdman, Phys. Rev. Lett. 84, 4337 (2000).

[7] M. B. Willemsen, M. P. van Exter, and J. P. Woerdman, Phys. Rev. A 60, 4105 (1999).

[8] M. P. van Exter, R. F. M. Hendriks, and J. P. Woerdman, Phys. Rev. A 57, 2080 (1998).

[9] M. P. van Exter, M. B. Willemsen, and J. P. Woerdman, Phys. Rev. A 58, 4191 (1998).
[10] V. A. Gaisyonok, E. V. Grigorieva, and S. A. Kashchenko, Opt. Commun. 124, 408 (1996).

[11] V. N. Chizhevsky, E. V. Grigorieva, and S. A. Kashchenko, Opt. Commun. 133, 189 (1997).

[12] R. S. Eisenberg, M. M. Klosek, and Z. Schuss, J. Chem. Phys. 102, 1767 (1995).

[13] R. S. Maier and D. L. Stein, Phys. Rev. Lett. 85, 1358 (2000).

[14] R. S. Maier and D. L. Stein, Phys. Rev. Lett. 71, 1783 (1993).

[15] R. S. Maier and D. L. Stein, J. Stat. Phys. 83, 291 (1996).

[16] R. S. Maier and D. L. Stein, Phys. Rev. Lett. 77, 4860 (1996).

[17] V. N. Smelyanskiy and M. I. Dykman, Phys. Rev. E 55, 2516 (1997)

[18] V. N. Smelyanskiy, M. I. Dykman, and R. S. Maier, Phys. Rev. E 55, 2369 (1997).

[19] M. I. Dykman, M. M. Millonas, and V. N. Smelyanskiy, Phys. Lett. A 195, 53 (1994).

[20] V. N. Smelyanskiy, M. I. Dykman, and B. Golding, Phys. Rev. Lett. 82, 3193 (1999).

[21] R. S. Maier and D. L. Stein, Phys. Rev. Lett. 86, 3942 (2001). 
[22] J. Lehmann, P. Reimann, and P. Hänggi, Phys. Rev. Lett. 84, 1639 (2000).

[23] J. Lehmann, P. Reimann, and P. Hänggi, Phys. Rev. E 62, 6282 (2000).

[24] A. Hamm and R. Graham, J. Stat. Phys. 66, 6891 (1992).

[25] R. Graham and T. Tel, Phys. Rev. Lett. 52, 9 (1984).

[26] R. Graham and T. Tel, Phys. Rev. A 31, 1109 (1985).

[27] R. Graham, in Noise in Nonlinear Dynamical Systems, edited by F. Moss and P. V. E. McClintock, (Cambridge University Press, Cambridge, U.K., 1989), vol. 1, pp. 225-278.

[28] H. R. Jauslin, Physica A 144, 179 (1987).

[29] M. V. Day, Stochastics 20, 121 (1987).

[30] P. Talkner, Z. Phys. B: Condens. Matter 68, 201 (1987).

[31] R. S. Maier and D. L. Stein, SIAM J. Appl. Math. 57, 752 (1997).

[32] M. I. Dykman, D. G. Luchinsky, P. V. E. McClintock, and V. N. Smelyanskiy, Phys. Rev. Lett. 77, 5229 (1996).

[33] V. N. Smelyanskiy, M. I. Dykman, H. Rabitz, and B. E. Vugmeister, Phys. Rev. Lett. 79, 3113 (1997).

[34] T. Naeh, B. Kłosek, M. M. Matkovsky, and Z. Schuss, SIAM J. Appl. Math. 50, 595 (1990).

[35] M. I. Dykman et al., Int. J. Bifurcation Chaos Appl. Sci. Eng. 8, 747 (1998).

[36] D. G. Luchinsky et al., Int. J. Bifurcation Chaos Appl. Sci. Eng. 12, 583 (2002).

[37] A. N. Silchenko, S. Beri, D. G. Luchinsky, and P. V. E. McClintock, Phys. Rev. Lett. 91, 174104 (2003).

[38] A. Silchenko, S. Beri, D. G. Luchinsky, and P. V. E. McClin- tock, Appl. Nonlinear Dyn. (Saratov) 11, 38 (2003).

[39] S. Kraut and U. Feudel, Phys. Rev. E 67, 015204(R) (2003).

[40] P. D. Beale, Phys. Rev. A 40, 3998 (1989).

[41] D. Ludwig, SIAM Rev. 17, 605 (1975).

[42] D. G. Luchinsky, P. V. E. McClintock, and M. I. Dykman, Rep. Prog. Phys. 61, 889 (1998).

[43] M. I. Dykman P. V. E. McClintock, V. N. Smelyanski, N. D. Stein, and N. G. Stocks, Phys. Rev. Lett. 68, 2718 (1992).

[44] M. Freidlin and A. D. Wentzel, Random Perturbations in Dynamical Systems (Springer, New York, 1984).

[45] V. I. Arnold, Mathematical Methods of Classical Mechanics (Springer-Verlag, Berlin, 1978).

[46] Some authors use for such a point the term focus. See for instance A. A. Andronov, E. A. Leontovich, I. I. Gordon, and A. G. Maier, Qualitative Theory of Second-Order Dynamical Systems (Wiley, New York, 1973).

[47] A nonrigorous definition of diffeomorphism is a transformation between two spaces that is invertible and differentiable, and whose inversion is also differentiable. The two spaces are said to be diffeomorph.

[48] B. van der Pol, Philos. Mag. 6, 763 (1928).

[49] M. Henon, Commun. Math. Phys. 50, 69 (1976).

[50] E. Ott, Chaos in Dynamical System (Cambridge University Press, Cambridge, U.K., 2002).

[51] P. Grassberger, J. Phys. A 22, 3283 (1989).

[52] S. W. McDonald, C. Grebogi, E. Ott, and J. A. Yorke, Physica D 17, 125 (1985).

[53] G. Julia, J. Math. Pures Appl. 8, 47 (1918). 